OPEN ACCESS

Edited by:

Bin Zhou,

Hunan University, China

Reviewed by: Wu Chen,

Southeast University, China Yujun $L i$,

Xi'an Jiaotong University, China Xiong Wu,

Xi'an Jiaotong University, China Qiang Yang,

Zhejiang University, China

${ }^{*}$ Correspondence: Ping Yang eppyang@scut.edu.cn

Specialty section: This article was submitted to Process and Energy Systems Engineering, a section of the journal

Frontiers in Energy Research

Received: 19 July 2021 Accepted: 30 August 2021 Published: 17 September 2021

Citation:

Li P, Zhang F, Ma X, Yao S, Zhong Z, Yang $P$, Zhao Z, Lai CS and Lai LL (2021) Multi-Time Scale Economic Optimization Dispatch of the Park Integrated Energy System.

Front. Energy Res. 9:743619. doi: 10.3389/fenrg.2021.743619

\section{Multi-Time Scale Economic Optimization Dispatch of the Park Integrated Energy System}

\author{
Peng $\mathrm{Li}^{1}$, Fan Zhang ${ }^{1}$, Xiyuan Ma ${ }^{1}$, Senjing Yao ${ }^{1}$, Zhuolin Zhong $^{2}$, Ping Yang ${ }^{2 *}$, Zhuoli Zhao ${ }^{3}$, \\ Chun Sing Lai ${ }^{4}$ and Loi Lei Lai ${ }^{3}$ \\ ${ }^{1}$ Digital Grid Research Institute of China Southern Power Grid, Guangzhou, China, ${ }^{2}$ Guangdong Key Laboratory of Clean Energy \\ Technology, South China University of Technology, Guangzhou, China, ${ }^{3}$ School of Automation, Guangdong University of \\ Technology, Guangzhou, China, ${ }^{4}$ Brunel Interdisciplinary Power Systems Research Centre, Department of Electronic and \\ Electrical Engineering, Brunel University London, London, United Kingdom
}

The park integrated energy system (PIES) plays an important role in realizing sustainable energy development and carbon neutral. Furthermore, its optimization dispatch can improve the energy utilization efficiency and reduce energy systems operation cost. However, the randomness and volatility of renewable energy and the instability of load all bring challenges to its optimal operation. An optimal dispatch framework of PIES is proposed, which constructs the operation models under three different time scales, including day-ahead, intra-day and real-time. Demand response is also divided into three levels considering its response characteristics and cost composition under different time scales. The example analysis shows that the multi-time scale optimization dispatch model can not only meet the supply and demand balance of PIES, diminish the fluctuation of renewable energy and flatten load curves, but also reduce the operation cost and improve the reliability of energy systems.

Keywords: integrated energy system, multi-time scale, optimization dispatch, renewable energy, demand response

\section{INTRODUCTION}

The park integrated energy system (PIES) is one of the main forms of distributed renewable energy utilization, which plays an important role in promoting the absorption of renewable energy and establishing a new power system under lower-carbon (Zahedi, 1994; Tang et al., 2018). Due to the fluctuation of renewable energy and the increase of load prediction error caused by the increase of forecast time scale, it is difficult to satisfy the balance of supply and demand through energy systems dispatch under a single time scale. Thus, the multi-time scale optimization dispatch of PIES is proposed. The dispatch plan is updated and adjusted according to the coordination of energy equipment and the use of more accurate forecast information of renewable energy and load, so as to ensure the economic operation, reduce the volatility of renewable energy and load, and improve the feasibility of the dispatch plan (Fan et al., 2021).

Many progresses have been made in the research on the coordinated planning of multiple types energy equipment in PIES and the improvement of overall operation economy. Literature (Zhang et al., 2019; Hui and Wei, 2020) constructed an integrated electricity-gas-thermal system planning model considering demand response, energy storage and energy conversion. Literature Jiang and Ai (2019) put forward an integrated energy system optimization operation model considering the new energy incentive mechanism to clarify the influence of different incentive mechanisms on the economic benefits. According to the quasi-dynamic characteristics of the thermal system, Literature 
Cen et al. (2020) established a building virtual energy storage model and an integrated energy utilization optimization model under the TOU price. Literature Lin and Fang (2019) established a regional integrated energy management system model based on multi-agent method to optimize operator profit and user cost. Literature Zeng et al. (2017) introduced a control optimization strategy considering economic performance and response performance to improve the multi-energy optimization of integrated energy system. Literature Zhang and Wei (2020) proposed a two-layer capacity allocation optimization method for IES based on the combined gas cooling, heating and power supply scheme.

However, the above literatures mainly studied the principle of economic optimal operation and the framework of system model. The integrated demand response is not considered or just regarded as a constraint condition of the model and the operation method and response strategy of the integrated demand response are lack of in-depth exploration. In Literature ( $\mathrm{Pu}$ et al., 2020), an optimal operation method of power system considering power demand response was proposed to transform the energy supply mode of port integrated energy system. Literature Lv et al. (2021) established an optimized operation model of IES and analyzed the flexibility of demand response under different coupling degrees. Literature Zhao et al. (2019a) introduced an electric-gas-thermal system and proposed a rolling operation optimization strategy considering the multienergy demand response and wind penetration. Literature Qi et al. (2020) proposed a two-layer collaborative optimization method of IES considering DR and ES. In Literature (Yu et al., 2020), a multi-energy flow calculation model and a dayahead optimal scheduling model for the power-gas-heat-cooling integrated energy system were established considering the integrated demand-side response, wind power output prediction and energy storage. Literature Wang C. et al. (2020) established a demand response model based on compensation mechanism to analyze the scheduling elasticity of different load types. Literature Wang J. et al. (2020) proposed a two-stage optimal scheduling model for integrated energy system from the perspective of user demand-side load control.

The time scale characteristics of each energy flow in the comprehensive energy are different. In terms of operation dispatch of integrated demand response, it is necessary to discuss the response speed of different energy flows to price change and external demand, analyze the influence of start-stop time and climbing speed of equipment on the complementarity of energy sources, study the time requirements of different energy flows on the balance between supply and demand (Duan et al., 2017). The response strategy and cost of load under different time scales also need to be studied. In Literature (Yang et al., 2020), a day-ahead and intra-day optimal scheduling model for integrated energy systems considering demand side response was established to suppress the intra-day fluctuations of renewable energy and loads. Literature Ouyang et al. (2019) constructed a multi-time scale optimization model with three different time response characteristics of electricity, heat and cold to optimize the economic operation of energy systems. Literature Xue et al. (2020) presented a real-time energy management method for integrated heating and power system based on approximate dynamic programming algorithm. Although the above studies introduced integrated demand response to improve the flexibility of load dispatch and increase the economic benefits of energy systems, they did not consider the uncertainty of load response at a small time scale and the impact on the operation dispatch of energy systems, so they could not meet the real-time adjustment requirements of energy systems under a small time scale.

To sum up, this paper shows the comparisons of related works in Table 1, and summarizes the advantages and disadvantages of several significant papers in Table 2 . Hence this paper proposes an economic optimization dispatch method for PIES based on multi-time scale demand response. Aiming at minimizing the operation cost of PIES, this paper constructs an optimization dispatch model under three-time scales, including day-ahead, intra-day and real-time. Based on the above model, this paper proposes a dispatch strategy and uses MATLAB to solve the mixed integer linear programming problem. Finally, the example analysis verifies the effectiveness and feasibility of the proposed method.

The key contributions of this paper are listed as follows.

1) Constructing a three-layer dispatch framework of PIES considering demand response, including the day-ahead, intra-day and real-time optimal dispatch.

2) Proposing the multi-time scale economic optimization strategy of PIES based on the multi-energy coordination and the effect of the fluctuation of renewable energy and load, including giving the objective functions, constraints and corresponding solution strategies in consideration of demand response.

3) Conducting case studies under a PIES scenario to verify the effectiveness of the proposed model and strategy, and analyzing the dispatch scheme and economic benefits of PIES under different time scales.

The organization of this study is as follows. PIES Dispatch Model introduces the structure and dispatch framework of PIES, and presents the dispatch model. Economic Optimal Dispatch of PIES Considering Demand Response proposes the dispatch strategies of PIES in consideration of demand response and solution scheme. Case Study describes the feasibility of PIES multi-time scale optimal dispatch based on the analysis of the calculation results. Conclusion summarizes the contributions and extensions of this paper.

\section{PIES DISPATCH MODEL}

\section{Structure Diagram of PIES}

According to the geographical factors and the characteristics of energy generation/transmission/distribution/use, the integrated energy system can be divided into trans-regional level, regional level, and user level, while PIES is the most typical representative of the regional integrated energy system (RIES). PIES takes active distribution network, hybrid energy storage and energy 
TABLE 1 | Comparison of related works ( $\sqrt{ }$ :Considered, - :Not considered).

Reference

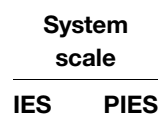

Time scale

\begin{tabular}{l} 
Demand \\
response \\
\hline PDR IDR
\end{tabular}

Energy flow

IES PIES

$\overline{\text { Day-ahead Intra-day Real-time }}$

$E / H / G$

$E / H / G / C$

Zhang et al. (2019); Wang et al. (2020a); Lv et al. (2021)

Jiang and Ai, (2019); Hui and Wei, (2020)

Lin and Fang, (2019); Cen et al. (2020)

Zeng et al. (2017)

Zhao et al. (2019a)

Yu et al. (2020)

Yang et al. (2020)

Ouyang et al. (2019)

Xue et al. (2020)

$\begin{array}{lll}\sqrt{ } & - & - \\ \sqrt{ } & - & \sqrt{ } \\ - & \sqrt{ } & - \\ \sqrt{ } & - & - \\ \sqrt{ } & - & \sqrt{ } \\ - & \sqrt{ } & \sqrt{ } \\ - & \sqrt{ } & \sqrt{ } \\ \sqrt{ } & - & \sqrt{ } \\ \sqrt{ } & - & \end{array}$

-
-
-
$\sqrt{ }$
$\sqrt{ }$
$\sqrt{ }$
$\sqrt{ }$
$\sqrt{ }$

$\begin{array}{ll}- & - \\ - & - \\ - & - \\ \sqrt{ } & - \\ - & - \\ \sqrt{ } & - \\ \sqrt{ } & \end{array}$

-
-
-
-
-
$\sqrt{ }$
$\sqrt{ }$

$\begin{array}{cccc}- & \sqrt{ } & \sqrt{ } & - \\ - & - & \sqrt{ } & - \\ \sqrt{ } & - & \sqrt{ } & - \\ - & \sqrt{ } & - & \sqrt{ } \\ - & \sqrt{ } & \sqrt{ } & - \\ - & \sqrt{ } & - & \sqrt{ } \\ \sqrt{ } & \sqrt{ } & - & \sqrt{ } \\ - & - & - & \sqrt{ } \\ - & - & \sqrt{ } & -\end{array}$

TABLE 2 | Comparison of significant works.

\begin{tabular}{|c|c|c|c|c|}
\hline Reference & $\begin{array}{l}\text { Multi-time } \\
\text { scale }\end{array}$ & $\begin{array}{l}\text { Energy } \\
\text { flow }\end{array}$ & Advantages & Disadvantages \\
\hline Zeng et al. (2017) & Yes & $E / H / G / C$ & $\begin{array}{l}\text { 1. Specific response time of different equipment } \\
\text { 2. Connection between economy and response } \\
\text { time }\end{array}$ & $\begin{array}{l}\text { 1. Lack of long-term dispatch } \\
\text { 2. Lack of analysis of multi-equipment response }\end{array}$ \\
\hline $\begin{array}{l}\text { Zhao et al. } \\
\text { (2019a) }\end{array}$ & Yes & $E / H / G$ & $\begin{array}{l}\text { 1. Rolling optimization } \\
\text { 2. Detailed demand response implementation } \\
\text { process }\end{array}$ & $\begin{array}{l}\text { 1. Limits of time scale } \\
\text { 2. Lack of cool flow }\end{array}$ \\
\hline Yu et al. (2020) & No & $\mathrm{E} / \mathrm{H} / \mathrm{G} / \mathrm{C}$ & $\begin{array}{l}\text { 1. Detailed demand response implementation } \\
\text { process } \\
\text { 2. Forecast output of renewable energy }\end{array}$ & Limits of time scale \\
\hline Yang et al. (2020) & Yes & $\mathrm{E} / \mathrm{H} / \mathrm{G} / \mathrm{C}$ & $\begin{array}{l}\text { 1. Rolling optimization } \\
\text { 2. Real-time scale }\end{array}$ & $\begin{array}{l}\text { No distinct characteristics of demand response under different } \\
\text { time scales }\end{array}$ \\
\hline
\end{tabular}

conversion technologies as the core, including intelligent distribution system, heating/cooling system, natural gas system and other multi-energy coupling networks (Zhang et al., 2021). Compared with the traditional integrated energy system, PIES has a shorter energy transmission distance, more accurate and fast energy system operation, and a higher degree of coupling between energy systems.

The structure of PIES is shown in Figure 1. Energy systems are coupled by four energy flows: electricity, gas, heating and cooling, and them contain a variety of energy production, conversion and storage equipment to meet different loads. However, only CCHP is selected as the energy coupling equipment in the system structure, which consists of gas turbine, waste heat boiler, absorption refrigerator, and electricity chiller. On one hand, for general energy systems, CCHP can not only undertake the full function of energy conversion (Zhao et al., 2018), but also is the most effective unit to improve the economic benefits (Ming et al., 2015; Zeng et al., 2017). On the other hand, this measure

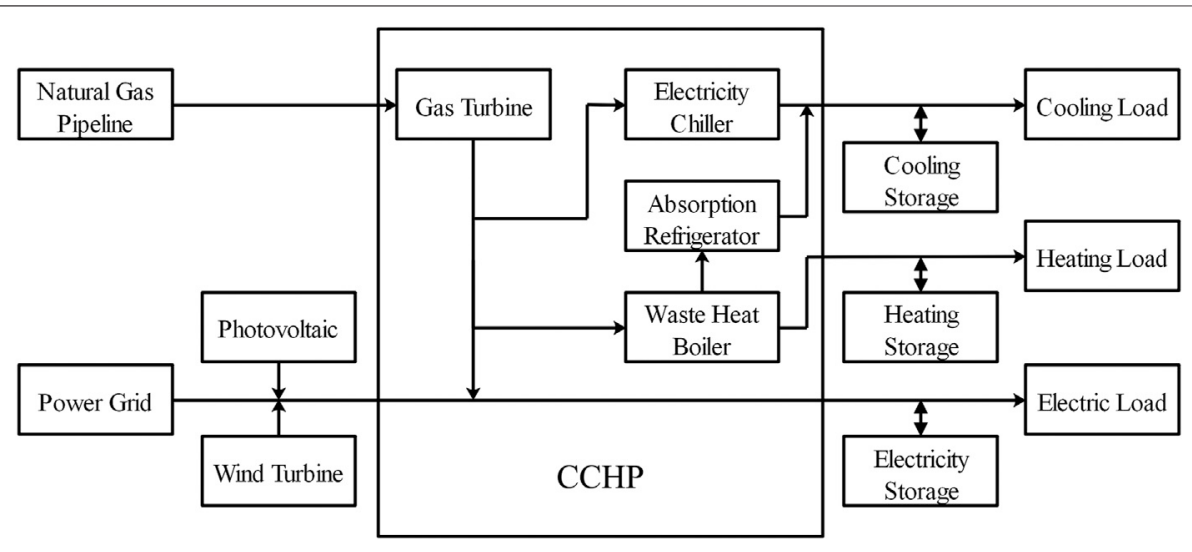

FIGURE 1 | Structure diagram of PIES. 


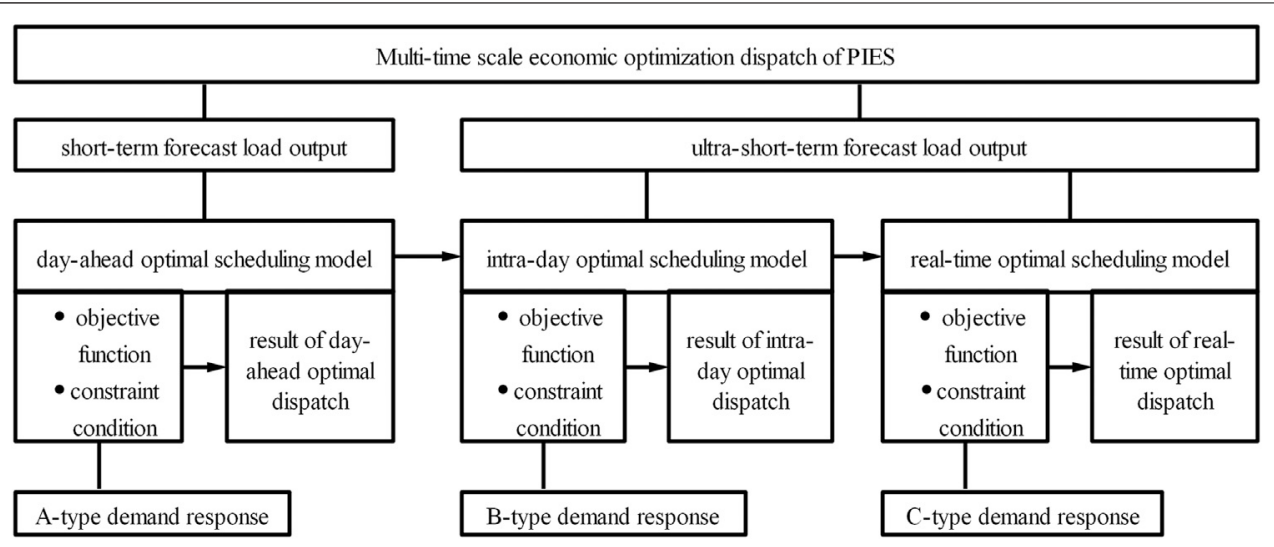

FIGURE 2 | Optimal dispatching framework of PIES.

can simplify the calculation, which can avoid discussing the calculation of the start-stop state of other energy conversion equipment and the impact of the start-stop cost on the economy. Since there are many studies on the steady state mathematical model of CCHP, this paper will not elaborate on it (Cheng et al., 2016; Zhao et al., 2018).

\section{Dispatch Framework of PIES}

In the optimization operation of PIES, day-ahead dispatch needs to formulate the operation plan of energy systems $24 \mathrm{~h}$ in advance, while intra-day and real-time dispatch should be scheduled according to energy production and coupling equipment output at various periods. Firstly, a day-ahead optimization dispatch can realize the optimal configuration under a long time scale and improve the economy of system operation. However, considering that the forecasting errors caused by renewable energy and load gradually decrease with the approaching of running time point (Drauz et al., 2018), dayahead dispatch cannot meet the actual operation requirements. Meanwhile, intra-day dispatch and real-time dispatch can make use of the forecasting information of load for dispatch under a short time scale, but they cannot consider the global coordination and collocation in the operation plan of energy systems.

On the other hand, integrated demand response faces the following problems in the multi-time scale optimization dispatch (Duan et al., 2017). For one thing, from the perspective of time scales, it is necessary to adapt to the prediction error caused by different time dimensions, the adjustment of resource scope and the difference of cost. For another, it is essential to adapt to the difference of demand response and loads with different characteristics when they participate in the power grid dispatching operation.

Based on the above problems, this paper proposes a combinatorial optimization dispatch framework for PIES, which consists of day-ahead optimal dispatch model, intra-day optimal dispatch model and real-time dispatch model, as shown in Figure 2. First, the day-ahead optimization dispatch plan is formulated every $24 \mathrm{~h}$ and the time scale is set as $1 \mathrm{~h}$. The interactive power between energy systems and power grid, the output of dispatchable energy equipment and flexible load response can be determined according to the short-term forecast output of day-ahead load and the predicted power of photovoltaic and wind turbines (Zhao et al., 2019b). Second, on the basis of day-ahead optimal dispatch, the intra-day optimization dispatch plan is made every $1 \mathrm{~h}$ to adjust the output of multiple equipment in the future $4 \mathrm{~h}$ and the time scale is set as $15 \mathrm{~min}$. Third, real-time dispatch takes into account the intraday optimization dispatch and makes a plan every $15 \mathrm{~min}$. It determines the next time scheduling plan according to ultrashort-term forecast load output.

There are three kinds of demand response in the optimal scheduling model of PIES, which are all incentive-oriented demand responses (IDR). According to the time of advance notification to users, the IDR is divided into three categories: 1 day in advance (A-type demand response), 4 hours in advance (B-type demand response), and 15 minutes in advance (C-type demand response).

\section{Dispatch Model of PIES}

This paper takes $24 \mathrm{~h}$ forecast power of load and renewable energy generation as the input of model, while dispatchable output of the combination of energy systems and equipment as the output of model, so as to construct an optimization dispatch model of PIES, which is expressed as follows:

$$
P^{\text {buy }}-P^{S O C}+P^{C C H P}+P^{D R}=P^{\text {load }}-\left(P^{W T}+P^{P V}\right)
$$

It is assumed that the dispatch plan of energy systems is divided into $n$ time periods. In terms of input, $P^{\text {load }}$ is a column vector of length $n$ that indicates forecasting load and its element $P_{i}^{\text {load }}$ indicates the load forecasting power in the $i$-th period. $P^{W T}$ and $P^{P V}$ are column vectors indicating prediction power of wind turbine and photoelectric and their length are both $n$. Their elements $P_{i}^{W T}$ and $P_{i}^{P V}$ indicate the predicted power output of wind turbine and photovoltaic in the $i$-th period.

In terms of output, energy purchase of energy systems mainly includes electricity and gas, so $P^{b u y}$ is a matrix of $n \times 2$, whose element $P_{i j}^{b u y}$ indicates the purchased power of the $j$-th type energy in the $i$-th period. The output of energy storage system $P^{S O C}$ includes 
three parts: electricity, heat and cool. Therefore, $P^{S O C}$ is a matrix of $n \times 3$, whose element $P_{i j}^{S O C}$ indicates the storage and discharge power of the $j$-th type energy storage in the $i$-th period. Here positive values indicate charging and negative values indicate discharging. $P^{C C H P}$ is an $n \times 3$ matrix indicating the power generation, heating and refrigeration of CCHP equipment (combined cooling heating and power) and its element $P_{i j}^{C C H P}$ indicates the output power of the $j$-th type energy generation in the $i$-th period. The length of load response vector $P^{D R}$ is $n$ and its element $P_{i}^{D R}$ indicates the dispatchable load of energy systems in the $i$-th period under the corresponding time scale.

According to Eq. 1, the dispatch model of PIES can realize optimal dispatch through reasonable arrangement of energy purchase, energy storage equipment, CCHP equipment, and dispatch strategies of flexible loads under the condition that the energy supply demand is different between the predicted output of energy systems load and that of the renewable energy.

\section{ECONOMIC OPTIMAL DISPATCH OF PIES CONSIDERING DEMAND RESPONSE}

\section{The Objective Function of Economic Optimal Dispatch}

The Objective Function of Day-Ahead Dispatch

In the day-ahead optimization dispatch model, this paper takes the cost of energy storage and CCHP equipment operation, energy purchase and load response as decision variables to solve the minimum operating cost of energy systems in the future $24 \mathrm{~h}$ considering the short-term forecast load:

$$
\begin{gathered}
\min F_{1}=F_{t}^{s, x}+F_{t}^{C C H P}+F_{t}^{\text {buy }}-F_{t}^{\text {sub }, A} \\
F_{t}^{s, x}=\left(U_{t}^{x, \text { in }} c^{x, \text { in }} P_{t}^{x}+U_{t}^{x, \text { out }} c^{x, \text { out }} P_{t}^{x}\right) \Delta T_{1} \\
F_{t}^{C C H P}=c^{C C H P} P_{t}^{C C H P} \Delta T_{1} \\
F_{t}^{\text {buy }}=F_{t}^{\text {grid }}+F_{t}^{\text {gas }} \\
F_{t}^{\text {grid }}=c_{t}^{\text {grid }} P_{t}^{\text {grid }} \Delta T_{1} \\
F_{t}^{\text {gas }}=c^{\text {gas }} G_{t}^{\text {gas }} \Delta T_{1} \\
F_{t}^{\text {sub,A }}=\eta^{\text {sub }} c^{\text {sub,A }} P_{t}^{D R, A} \Delta T_{1}
\end{gathered}
$$

where $F_{1}$ indicates the expected total cost of the day-ahead system; $F_{t}^{s, x}, F_{t}^{C C H P}, F_{t}^{b u y}$ and $F_{t}^{s u b, A}$ respectively indicate the operation and maintenance cost of energy storage equipment, the operation and maintenance cost of CCHP equipment, energy purchase cost and A-type demand response compensation in the $t$-th period; $F_{t}^{\text {grid }}$ and $F_{t}^{g a s}$ indicate electricity purchase cost and gas purchase cost. $c^{x, \text { in }}$ and $c^{x, \text { out }}$ indicate charge and discharge maintenance price coefficients of energy storage equipment; $c^{C C H P}$ indicates the price coefficient of CCHP equipment operation and maintenance; $c_{t}^{\text {grid }}$ and $c^{g a s}$ indicate the electricity price purchased from power grid in the $t$-th period and unit natural gas price; $c^{s u b, A}$ indicates the subsidy standard of A-type demand response. $P_{t}^{x}$ indicates the charge and discharge power of the energy storage equipment in the $t$-th period, in which charging is positive and discharging is negative; $P_{t}^{g r i d}$ and $G_{t}^{g a s}$ indicate the electricity purchase power and the gas purchase capacity in the $t$-th period; $P_{t}^{C C H P}$ indicates the electricity power of CCHP equipment; $P_{t}^{D R, A}$ indicates the regulation power of A-type demand response. $\eta^{\text {sub }}$ indicates the response coefficient of demand response. $U_{t}^{x, \text { in }}$ and $U_{t}^{x, \text { out }}$ are $0-1$ variables, which indicate the charging and discharging states of the energy storage equipment. $x$ indicates energy type and $x$ is a member of the set $Z$, which contains three elements: $E, H$ and $C$, respectively indicating electricity, heat and cool energy.

\section{The Objective Function of Intra-day Dispatch}

If intra-day dispatch plan is re-formulated in each period considering the day-ahead dispatch, there may be a large difference between them (Zhao et al., 2021), which obviously does not satisfy the actual operation requirements. Therefore, intra-day optimization dispatch adjusts the output of each equipment every $1 \mathrm{~h}$ on the basis of day-ahead optimization dispatch, so as to minimize the total adjustment cost of energy systems in the next $4 \mathrm{~h}$ :

$$
\begin{gathered}
\min F_{2}=\Delta F_{1,2}^{s, x}+\Delta F_{1,2}^{C C H P}+\Delta F_{1,2}^{b u y}-F_{t}^{s u b, B} \\
\Delta F_{1,2}^{s, x}=F_{t, 1}^{s, x}-F_{t, 2}^{s, x} \\
\Delta F_{1,2}^{C C H P}=F_{t, 1}^{C C H P}-F_{t, 2}^{C C H P} \\
\Delta F_{1,2}^{b u y}=F_{t, 1}^{g r i d}-F_{t, 2}^{g r i d}+F_{t, 1}^{g a s}-F_{t, 2}^{g a s} \\
F_{t}^{s u b, B}=\eta^{s u b} c^{s u b, B} P_{t}^{D R, B} \Delta T_{2}
\end{gathered}
$$

where $F_{2}$ indicates the total adjustment cost of the intra-day system; $\Delta F_{1,2}^{s, x}, \Delta F_{1,2}^{C C H P}, \Delta F_{1,2}^{b u y}$ indicate the adjustment costs of energy storage equipment, CCHP equipment and energy purchasing plan of PIES between day-ahead and intra-day schedules in the $t$-th period; $F_{t}^{s u b, B}$ indicates B-type demand response compensation; $c^{\text {sub,B }}$ indicates the subsidy standard of B-type demand response; $P_{t}^{D R, B}$ indicates the regulation power of B-type demand response; subscripts 1 and 2 indicate day-ahead dispatch and intra-day dispatch.

\section{The Objective Function of Real-Time Dispatch}

Real-time dispatch adjusts the output of each equipment every $15 \mathrm{~min}$ at the next moment considering the ultra-short-term predicted output of load on the basis of intra-day optimization dispatch, so to minimize the adjustment cost in a single period of PIES as:

$$
\min F_{3}=\Delta F_{2,3}^{s, x}+\Delta F_{2,3}^{C C H P}+\Delta F_{2,3}^{b u y}-F_{t}^{s u b, C}
$$

where $F_{3}$ indicates the total adjustment cost of the real-time system; $\Delta F_{2,3}^{s, x}, \Delta F_{2,3}^{C C H P}$ and $\Delta F_{2,3}^{b u y}$ indicate the adjustment costs of energy storage equipment, CCHP equipment and energy purchasing plan of PIES between intra-day and real-time schedules in the $t$-th period; $F_{t}^{s u b, C}$ indicates C-type demand response compensation; subscripts 2 and 3 indicate intra-day dispatch and real-time dispatch.

\section{The Constraint Condition of Economic Optimal Dispatch System Power Equilibrium Constraint}

$$
P_{t}^{g r i d}+P_{t}^{P V}+P_{t}^{W T}+P_{t}^{C C H P}=P_{t}^{E S}+P_{t}^{L D}+P_{t}^{D R, A}
$$


where $P_{t}^{P V}$ and $P_{t}^{W T}$ indicate the power generation of photovoltaic and wind turbine in the $t$-th period; $P_{t}^{E S}$ indicates the charge and discharge power of the storage device, in which charging is positive and discharging is negative; $P_{t}^{D R, A}$ is positive when the load is transferred in and negative when the load is transferred out.

\section{System Thermal Equilibrium Constraint}

$$
H_{t}^{C C H P}-P_{t}^{H S}=H_{t}^{L D} / \eta_{\text {heat }}
$$

where $H_{t}^{C C H P}$ indicates the heating power of CCHP equipment; $P_{t}^{H S}$ indicates the charge and discharge power of heat storage equipment, in which charging is positive and discharging is negative; $H_{t}^{L D}$ indicates the thermal load of energy systems in the $t$-th period; $\eta_{\text {heat }}$ indicates the ratio of heat energy utilization in heating grid.

\section{System Cool Equilibrium Constraint}

$$
C_{t}^{C C H P}-P_{t}^{C S}=C_{t}^{L D} / \eta_{\text {cool }}
$$

where $C_{t}^{C C H P}$ indicates the refrigeration power of CCHP equipment; $P_{t}^{C S}$ indicates the charge and discharge power of cool storage equipment, in which charging is positive and discharging is negative; $C_{t}^{L D}$ indicates the cool load of energy systems in the $t$-th period; $\eta_{\text {cool }}$ indicates the ratio of cool energy utilization in cooling grid.

\section{Energy Storage Equipment Constraint}

$$
\begin{gathered}
S_{t}^{x}=\left(1-\eta_{s, x}\right) S_{t-1}^{x}+P_{t}^{x} \Delta T_{1} \\
S^{x, \min } \leq S_{t}^{x} \leq S^{x, \max } \\
S_{0}^{x}=S_{T}^{x} \\
P^{x, \min } \leq P_{t}^{x} \leq P^{x, \max }
\end{gathered}
$$

where $S_{t}^{x}$ indicates the capacity of various energy storage devices in the $t$-th period; $S^{x, \min }$ and $S^{x, \max }$ indicate the upper and lower limits of the capacity of energy storage equipment; $\eta_{s, x}$ indicates the self-consumption rate of energy storage equipment; $S_{0}^{x}$ and $S_{T}^{x}$ indicate the energy storage capacity at the beginning and the end of dispatch.

\section{CCHP Operating Constraint}

$$
\begin{aligned}
P^{C C H P, \min } & \leq P_{t}^{C C H P} \leq P^{C C H P, \max } \\
H^{C C H P, \min } & \leq H_{t}^{C C H P} \leq H^{C C H P, \max } \\
C^{C C H P, \min } & \leq C_{t}^{C C H P} \leq C^{C C H P, \max }
\end{aligned}
$$

where $P^{C C H P, \min }$ and $P^{C C H P, \max }$ indicate the minimum and maximum generating power of CCHP equipment; $H^{\mathrm{CCHP} \text {, min }}$ and $H^{C C H P, \max }$ indicate the minimum and maximum heating power of CCHP equipment; $C^{C C H P, \min }$ and $C^{C C H P, \max }$ indicate the minimum and maximum refrigeration power of $\mathrm{CCHP}$ equipment.

\section{Dispatch Plan Deviation Constraint}

$$
\begin{gathered}
P_{t, 1}^{s, x}-P_{t, 2}^{s, x} \leq \mu P^{x, \max }, P_{t, 2}^{s, x}-P_{t, 3}^{s, x} \leq \mu P^{x, \max }, x \in Z \\
P_{t, 1}^{C C H P}-P_{t, 2}^{C C H P} \leq \mu P^{C C H P, \max }, P_{t, 2}^{C C H P}-P_{t, 3}^{C C H P} \leq \mu P^{C C H P, \max }
\end{gathered}
$$

where $\mu$ indicates the constraint constant; subscripts 1,2 and 3 indicate the day-ahead dispatch, intra-day dispatch and real-time dispatch. The deviation constraint is only used for intra-day and real-time dispatch.

\section{Multi-Time Scale Economic Optimization Dispatching Strategy for PIES}

In order to solve the optimal dispatch scheme of PIES, this paper schedules the cost coefficient and flexible load based on demand response under different time scales and the establishment of objective functions and constraint conditions in day-ahead, intraday and real-time dispatch. The specific solution process is shown in Figure 3.

In the above solving process, the multi-time scale economic optimal dispatch strategy of PIES constitutes the mixed integer linear programming (MILP) problem. Based on MATLAB software, this paper calls the CPLEX solver in the YALMIP installation package to solve the above MILP problem.

\section{CASE STUDY}

\section{Setting}

In order to verify the effectiveness of the multi-time scale optimization dispatch strategy of PIES, two typical cases are designed to simulate the multi-constraint dynamic linear programming problem including CCHP equipment and energy storage equipment.

This paper selects a PIES for analysis, and the main basic parameters are shown in Table 3. The unit price of natural gas $c^{\text {gas }}$ is 2.4 yuan $/ \mathrm{m}^{3}$, the low calorific value of natural gas is $36 \mathrm{MJ} / \mathrm{m}^{3}$ and the time-of-use electricity price is as follows: 0.821 yuan/ $\mathrm{kWh}$ in peak period, 0.485 yuan/ $\mathrm{kWh}$ in normal period and 0.368 yuan/ $\mathrm{kWh}$ in valley period; the operating range of the energy storage equipment is [5\%,95\%] and the initial capacity is $50 \%$; the proportion of thermal energy utilization $\eta_{\text {heat }}$ is 0.9 and the proportion of cool energy utilization $\eta_{\text {cool }}$ is 0.9 ; the maximum capacity that can be invoked for A-type, B-type and C-type demand response shall not exceed $6 \%$ of the maximum of electric load. The response coefficient and subsidy standard of demand response are shown in Table 4.

\section{Case Analysis}

\section{Typical Day in Summer}

This paper analyzes the daily output of each equipment on a typical day in summer. The power, heating and cooling load, and renewable energy curves of a typical day in summer are shown in Figure 4.

\section{Day-Ahead Optimization Operation Result and Analysis} According to the above example parameters, this paper runs the day-ahead optimal dispatch model of PIES and obtains the 


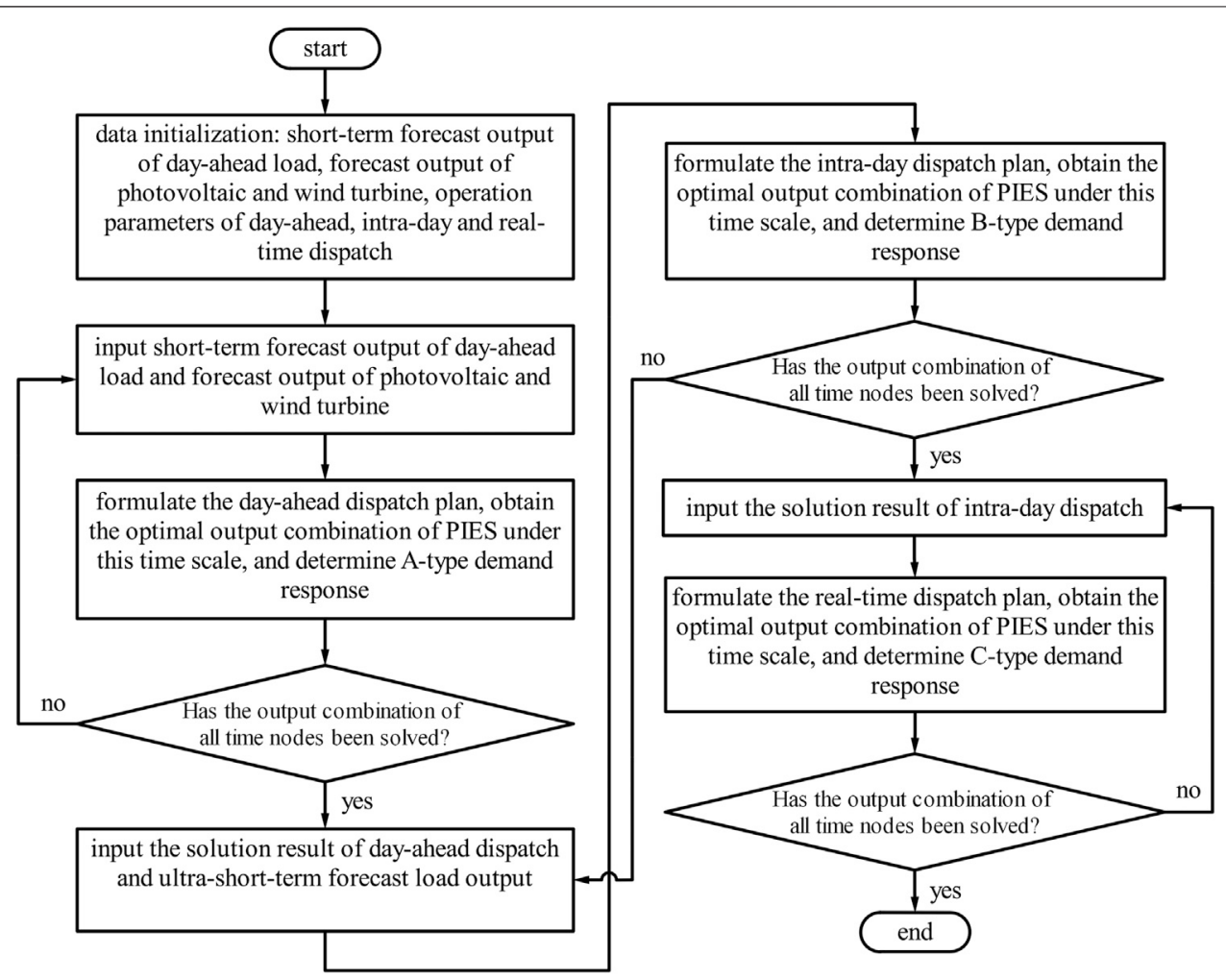

FIGURE 3 | The optimal dispatch solution process of PIES.

TABLE 3 | Basic performance parameters of the equipment.

\section{Equipment}

Operation and Maintenance

Cost (yuan/MWh)

Minimum Power (MW)

Maximum Power (MW)

Interconnection Capacity (MW)

Other Parameters

\section{CCHP}

0.04

0

100

Thermoelectric Ratio 1.85, Power Generation Efficiency 30\%, Heating Efficiency 85\%, Refrigeration Performance Coefficient 1.5

\section{E-storage}

0.009

$-3$

3

15

Self-discharge

Coefficient 0.02
H-storage

0.022

$-3$

3

Self-loss

Coefficient 0.015
C-storage

0.022

$-3$

3

25

Self-loss

Coefficient 0.015

TABLE 4 | Subsidies standard of load response.

Type of demand response

A-type Demand Response

B-type Demand Response

C-type Demand Response
Response coefficient $\eta^{\text {sub }}$

Period

Peak Period

Normal Period

Valley Period

Peak Period

Normal Period

Valley Period

Peak Period

Normal Period

Valley Period
Subsidy standard $c^{\text {sub }}$ (yuan/kWh) 


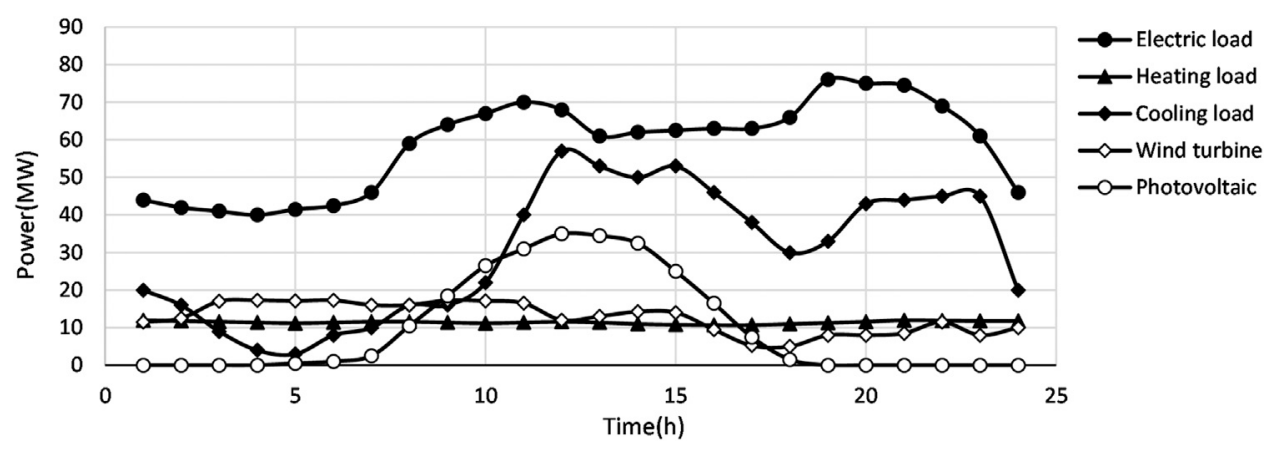

FIGURE 4 | Power, heating and cooling load, and renewable energy curves of a typical day in summer.

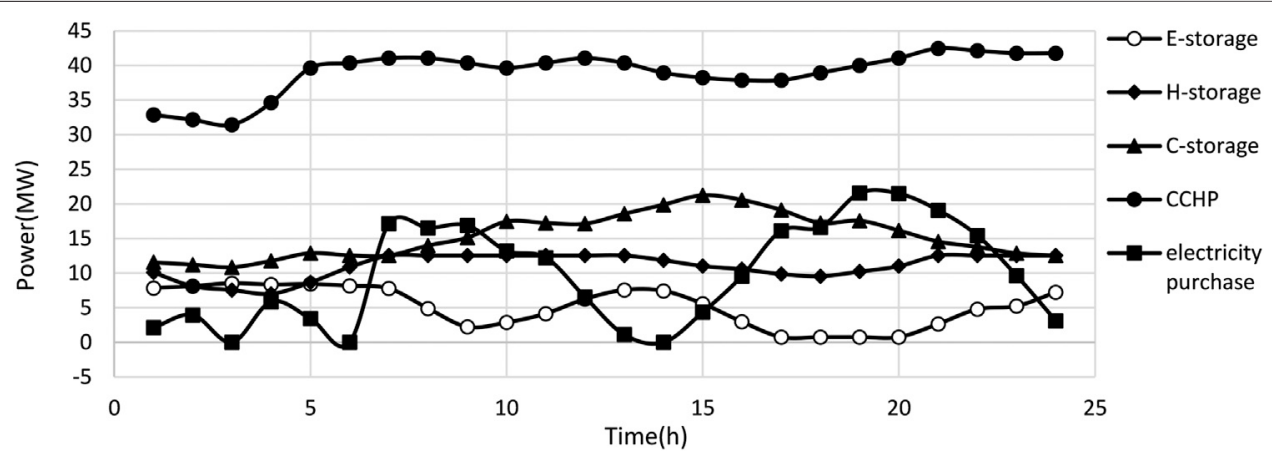

FIGURE 5 | Day-ahead optimization dispatch plan of a typical day in summer.

day-ahead optimal dispatch scheme on a typical day in summer, as shown in Figure 5.

It can be seen from Figure 5 that the day-ahead optimized operation plan can meet the balance between supply and demand of electricity, heat and cool load by reasonably arranging the output of each equipment.

When the electricity price is at the valley from 23:00 to 07:00 the next day, the electrical load is low and the thermal load and cool load are mainly powered by CCHP equipment. The electricity generated is enough to satisfy the demand of electrical load, so the electricity is less purchased and the storaging energy of electric storage equipment increases steadily.

Then the electrical load of energy systems reaches the first peak between 7:00 and 11:00. PIES increases the purchased electric power, adjusts the storage equipment to discharge and improves the storaging energy of cool storage equipment.

From 11:00 to 15:00, the first peak of electrical load ends and power generation of photovoltaic and wind turbine gradually rises, so the power supply of energy systems is relatively abundant. System reduces power purchase, while the energy storage of the electric storage equipment increases. Because of the continuous increase of cool load, CCHP equipment can only increase the output to maintain the cool balance, causing the cool storage energy further increases.

During 15:00 to 23:00, PIES ushers in the second peak of electrical load and increases the purchased electric power and adjusts the storage equipment for discharging. At about 19:00, the storage equipment consumes up the stored electric energy, so energy systems increase the purchased electric power again. As the cool load of energy systems also appears the second peak and the output of CCHP equipment changes little, energy systems arrange the cooling storage equipment to discharge energy to satisfy the cooling demand.

\section{Typical Day in Winter}

This paper analyzes the daily output of each equipment on a typical day in winter. The power, heating and cooling load, and renewable energy curves of a typical day in winter are shown in Figure 6.

\section{Day-Ahead Optimization Operation Result and Analysis}

According to the above example parameters, this paper runs the day-ahead optimal dispatch model of PIES and obtains the dayahead optimal dispatch scheme on a typical day in winter, as shown in Figure 7.

When the electricity price is at the valley from 22:00 to 07 : 00 the next day, the power load is low while the thermal load is keeping at a high level. In order to meet the heating demand, the electricity generated by CCHP exceeds the power load, so that energy systems sell electricity, and electric energy storage increases. On the contrary, thermal energy storage is decreasing. 


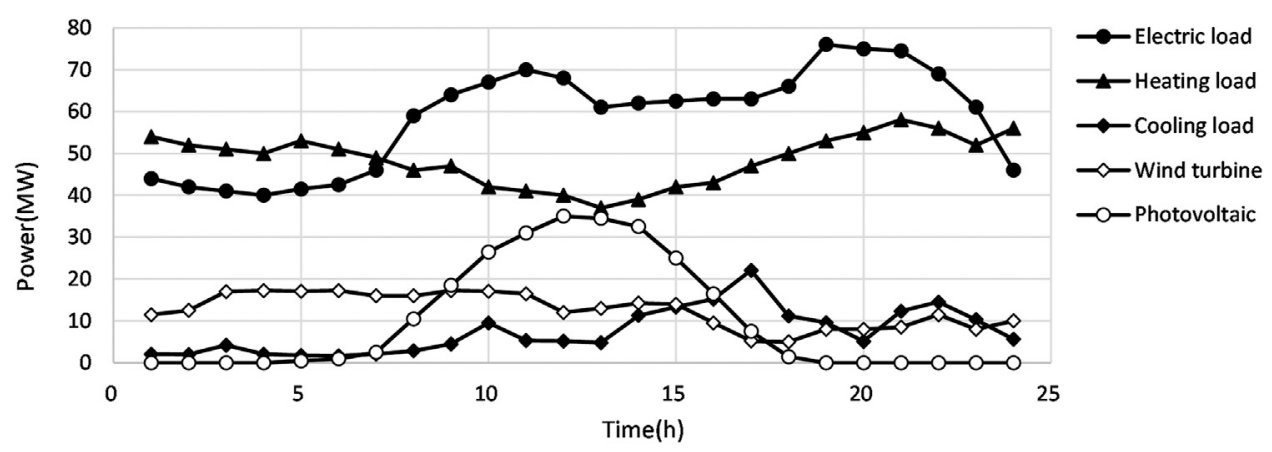

FIGURE 6 | Power, heating and cooling load, and renewable energy curves of a typical day in winter.

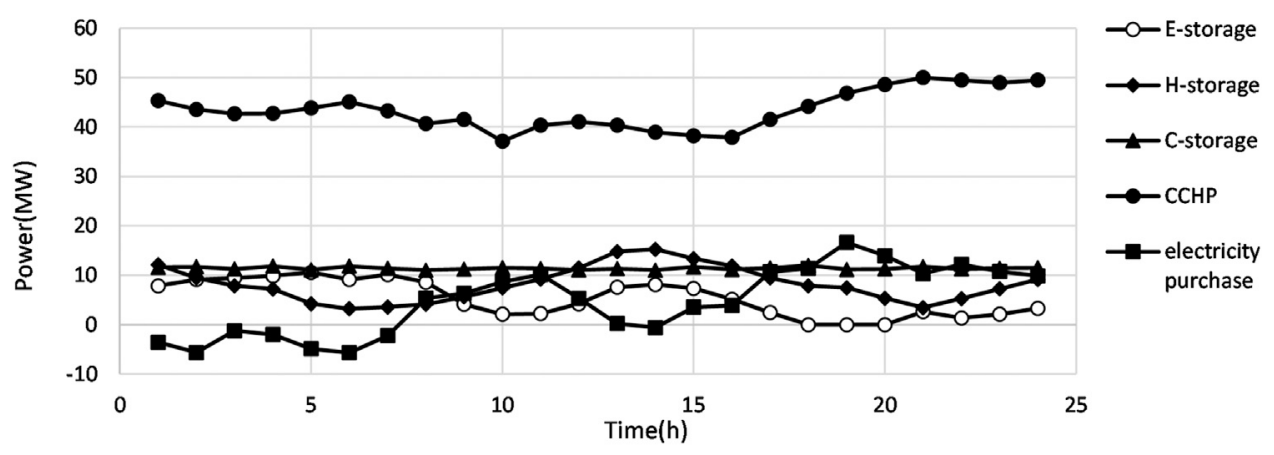

FIGURE 7 | Day-ahead optimization dispatch plan of a typical day in winter.

When it comes to the first peak from 7:00 to 11:00, as the heating load decreases, the output of CCHP also decreases. The insufficient power generation is supplemented by electric energy storage and purchased power.

From 11:00 to 15:00, the first peak of electrical load ends. During this period, the thermal load continues to decrease and reaches the lowest value at 13:00, while the output of CCHP remains basically unchanged in order to meet the power supply demand. As a result, thermal and electric energy storage increases.

When it comes to the second peak from 15:00 to 22:00, PIES increases the purchased electric power and adjusts the storage equipment for discharging. CCHP reaches maximum capacity at 21:00 and lasts for 2 hours. At the same time, the thermal load also reaches the peak, and the thermal energy storage has to operate at maximum discharging power.

In particular, cooling energy storage remains roughly the same regardless of how CCHP output changes. It is possible that energy systems release excess cooling energy in order to reduce the maintenance cost of the cooling storage equipment, when the output of CCHP is sufficient to satisfy the cooling load demand.

\section{Comparative Analysis of Day-Ahead, Intra-day and Real-Time Optimal Dispatch}

This paper substitutes the result of day-ahead optimization dispatch into the intra-day optimization dispatch model and the intra-day optimization dispatch plan can be obtained through calculation. Then this paper substitutes the intra-day dispatch result into the real-time dispatch model and also obtains the real-time dispatch result. Figure 8; Figure 9; Figure 10 show the curves of purchasing power from electric grid, gas purchasing capacity and demand response under multi-time scale.

As it can be seen from Figure 8, after the adjustment of intraday and real-time dispatch, the interactive power curve between energy systems and power grid fluctuates with the change of electricity price. The electricity purchase decreases during the peak period and the decrease are more obvious at the second peak period. It can be seen from Figure 9 that, in the peak period of electrical load, the gas purchase volume of energy systems under intra-day and real-time dispatching is improved compared with day-ahead dispatching. This is because electricity price is higher than the price of natural gas at the same energy consumption in the peak period, so adding power from CCHP equipment is more cost-effective than purchasing electricity.

As it can be seen from Figure 10, A-type demand response has a good function of peak clipping and valley filling, reducing the load in the peak period and increasing the load in the valley period. B-type demand response also has the function of peak clipping and valley filling, but the effect is weakened compared with A-type. C-type demand response has a small capacity and fluctuates, mainly because real-time dispatching needs to adjust the load response continuously in a short time to smooth down 

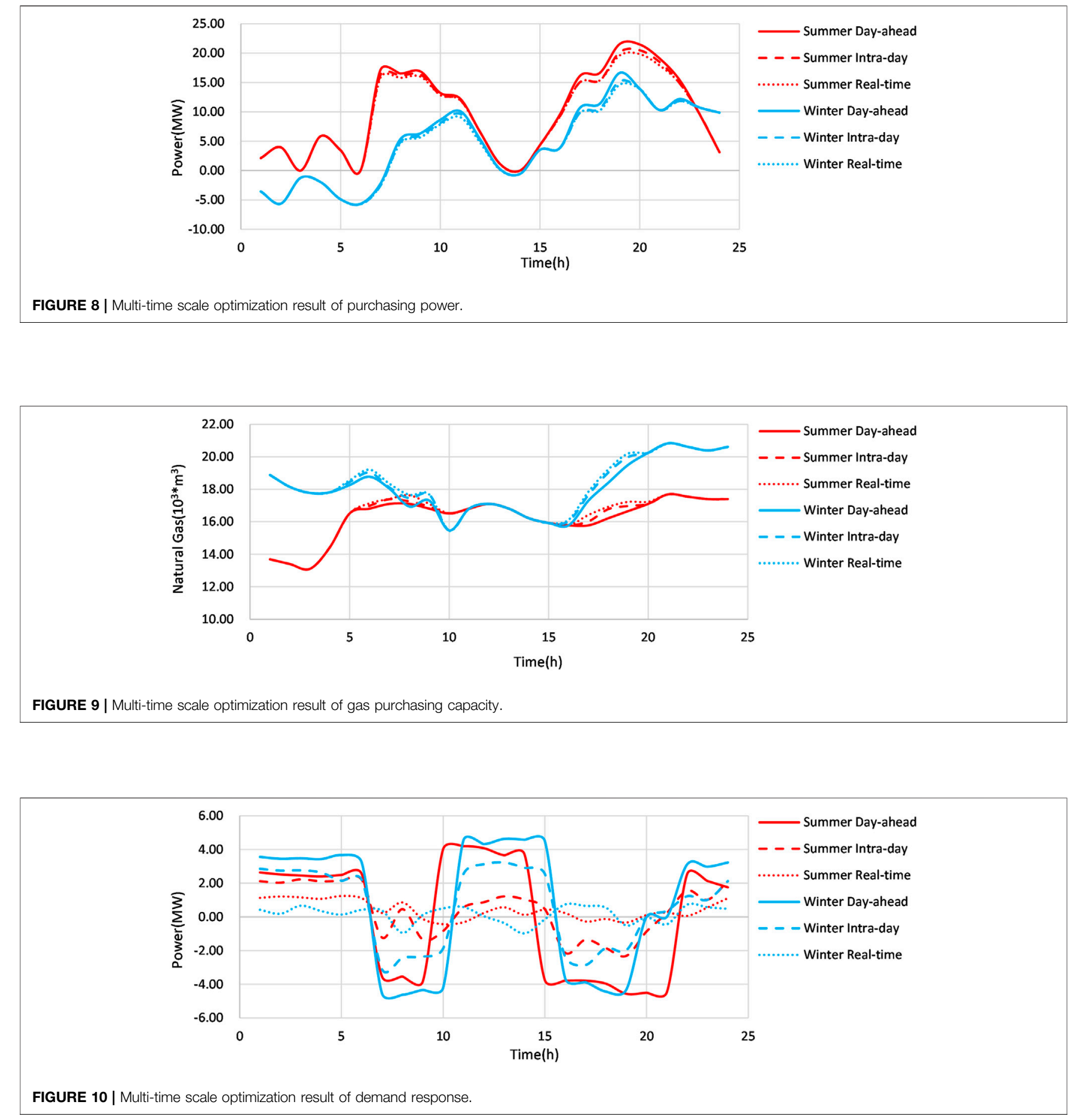

TABLE 5 | Comparison of operating costs under different dispatch schemes.

\begin{tabular}{llccc}
\hline Scenario & Dispatch scheme & Electricity purchase cost & Gas purchase cost & Total operating cost \\
\hline Summer & Day-ahead dispatch & 149670.8 & 935640.1 & 1112456.1 \\
\multirow{2}{*}{ Winter } & Multi-time dispatch & 122856.1 & 941040.7 & 1087654.4 \\
& Day-ahead dispatch & 128563.2 & 1041456.3 & 1210339.7 \\
& Multi-time dispatch & 101345.3 & 1048756.6 & 1185527.8
\end{tabular}


the fluctuation of load and renewable energy output, so as to ensure the power balance of energy systems. However, it should be noted in particular that, since the output of CCHP reaches its maximum at about 21:00 on a typical day in winter, the demand response has no ability to adjust downward.

\section{Cost Comparison of Optimal Dispatch of PIES}

Table 5 shows the Comparison of operating costs under different dispatch schemes. As it can be seen from Table 5, compared with day-ahead dispatch, the operation cost of multitime dispatch decreases by $2.23 \%$ in summer and $2.05 \%$ in winter. The above result verifies that the multi-time scale economic optimization dispatch of PIES considering the demand response can reduce the operation cost and improve economy efficiency.

\section{CONCLUSION}

In order to further improve the operation economy of PIES, this paper puts forward an economic optimization dispatch strategy under multiple time scales. Then energy systems optimize the dispatch plan by arranging the scheduling strategy of energy purchase, coupling energy equipment and flexible load reasonably. Finally, this paper analyzes the multi-time scale optimal dispatch results and economic benefits of energy systems. The specific contributions are as follows:

1) Adding the demand response conforming to different time scales into the optimization dispatch plan of PIES, which can realize the effect of peak clipping and valley filling, flatten the load curve, and reduce the operation cost of energy systems.

2) Scheduling electricity, heat and cool loads on different time scales by using the three-layer optimal dispatch model which is composed of day-ahead, intra-day and real-time, so that energy systems can balance the supply and demand relationship and ensure the stable operation.

Meanwhile, in view of the problems reflected in the research, this paper considers from the following aspects to further expand:

\section{REFERENCES}

Cen, Z., Yu, K., Chen, C., Chen, X., Dong, Z., and Gan, L. (2020). "Research on Optimal Operation of Regional Integrated Energy System Considering Virtual Energy Storage Characteristics of Buildings," in 2020 12th IEEE PES Asia-Pacific Power and Energy Engineering Conference (APPEEC) (IEEE), 1-5. doi:10.1109/ APPEEC48164.2020.9220542

Drauz, S. R., Spalthoff, C., Würtenberg, M., Kneikse, T. M., and Braun, M. (2018). “A Modular Approach for Co-simulations of Integrated Multi-Energy Systems: Coupling Multi-Energy Grids in Existing Environments of Grid Planning \& Operation Tools," in 2018 Workshop on Modeling and
1) This paper only formulates the response strategy of electric load, without considering the demand response of cooling and heating load.

2) This article will consider coupling other types of energy conversion equipment, including gas Boiler, Power to Gas, and others. Moreover, when CCHP reaches the maximum rated output, the demand response loses the negative adjustment ability. It is considered to add equipment with repetitive functions in CCHP to make up for the deficiency of $\mathrm{CCHP}$ capacity. This paper will also consider using gas storage equipment in the future.

3) This paper does not consider the technical characteristics and cost composition of energy transmission and conversion in different PIES, such as industrial PIES, residential PIES and business PIES. It is necessary to verify whether the model is applicable to different types of energy systems.

\section{DATA AVAILABILITY STATEMENT}

The original contributions presented in the study are included in the article/Supplementary Material, further inquiries can be directed to the corresponding author.

\section{AUTHOR CONTRIBUTIONS}

PL conceptualized the study, contributed to the study methodology, and wrote the original draft. FZ contributed to the study methodology, data curation and investigation. XM contributed to data analysis and investigation. SY contributed to software and formal analysis. ZLZ contributed to investigation and writing-original draft. PY contributed to supervision and writing-review \& editing. ZLZ contributed to investigation and writing-review \& editing. CSL contributed to data curation. LLL contributed to writing-review \& editing. All authors have read and agreed to the published version of the manuscript.

\section{FUNDING}

This work was supported by the Research Project of Digital Grid Research Institute, China Southern Power Grid under Grant YTYZW20010.

Simulation of Cyber-Physical Energy Systems (MSCPES) (IEEE), 1-6. doi:10.1109/MSCPES.2018.8405395

Duan, M., Shao, X., Cui, G., Zheng, A., Tong, G., Wu, G., et al. (2017). "Rolling Dispatch of Demand Response Resources Considering Renewable Energy Accommodation," in 2017 4th International Conference on Systems and Informatics (ICSAI) (IEEE), 358-362. doi:10.1109/ICSAI.2017.8248318

Fan, H., Yu, Z., Xia, S., and Li, X. (2021). Review on Coordinated Planning of Source-Network-Load-Storage for Integrated Energy Systems. Front. Energ. Res. 9, 138. doi:10.3389/fenrg.2021.641158

Hui, H., and Wei, L. (2020). "Modeling and Optimal Operation of Urban Integrated Energy System Considering Multiple Sub-systems," in 2020 10th International Conference on Power and Energy Systems (ICPES) (Chengdu, China: IEEE), 564-568. doi:10.1109/ICPES51309.2020.9349740 
Jiang, C., and Ai, X. (2019). "Study on Optimal Operation of Integrated Energy System Considering New Energy Incentive Mechanism," in 2019 IEEE 3rd Conference on Energy Internet and Energy System Integration (EI2) (IEEE), 301-306. doi:10.1109/EI247390.2019.9062262

Lin Cheng, L., Chen Liu, C., Renle Huang, R., and Hongtao Li, H. (2016). "An Optimal Operating Strategy for CCHP in Multi-Energy Carrier System," in 2016 IEEE Power and Energy Society General Meeting (PESGM) (IEEE), 1-5. doi:10.1109/PESGM.2016.7741902

Lin, R., and Fang, F. (2019). "Energy Management Method on Integrated Energy System Based on Multi-Agent Game," in 2019 International Conference on Sensing, Diagnostics, Prognostics, and Control (SDPC) (IEEE), 564-570. doi:10.1109/SDPC.2019.00107

Lv, H., Wang, Y., Dong, X., Jiang, F., Wang, C., and Zhang, Z. (2021). "Optimization Scheduling of Integrated Energy System Considering Demand Response and Coupling Degree," in 2021 IEEE/IAS 57th Industrial and Commercial Power Systems Technical Conference (I CPS) (IEEE), 1-8. doi:10.1109/ICPS51807.2021.9416612

Ming, Z., Qiqi, Q., Haojing, W., Yuming, G., Liang, G., Jian, Z., et al. (2015). "Economy Benefit Comparison of CCHP System and Conventional Separate Supply System," in 2015 8th International Conference on Intelligent Computation Technology and Automation (ICICTA) (Nanchang, China: IEEE), 402-406. doi:10.1109/ICICTA.2015.107

Ouyang, B., Yuan, Z., Qu, L., and Lu, C. (2019). "Research on Optimal Operation of Multi-Time Scale Cold-Thermal-Electric Integrated Energy System," in 2019 IEEE PES Asia-Pacific Power and Energy Engineering Conference (APPEEC) (IEEE), 1-5. doi:10.1109/APPEEC45492.2019.8994379

$\mathrm{Pu}$, Y., Chen, W., Zhang, R., and Liu, H. (2020). "Optimal Operation Strategy of Port Integrated Energy System Considering Demand Response," in 2020 IEEE 4th Conference on Energy Internet and Energy System Integration (EI2) (IEEE), 518-523. doi:10.1109/EI250167.2020.9346904

Qi, X., Xiong, H., Xiao, H., and Pei, W. (2020). "Coordinated Optimization of Integrated Energy System Considering Demand Response and Energy Storage," in 2020 23rd International Conference on Electrical Machines and Systems (ICEMS) (IEEE), 478-482. doi:10.23919/ICEMS50442.2020.9291056

Tang, F., Dai, H., Chen, X., and Zhao, Y. (2018). "Integrated Energy Technology and Service Trend in China: Investigation and Analysis on over 1800 Development Zones in Different Regions," in 2018 IEEE 2nd International Electrical and Energy Conference (CIEEC) (IEEE), 428-433. doi:10.1109/ CIEEC.2018.8745735

Wang, C., Chen, S., Mei, S., Chen, R., and Yu, H. (2020a). Optimal Scheduling for Integrated Energy System Considering Scheduling Elasticity of Electric and Thermal Loads. IEEE Access 8, 202933-202945. doi:10.1109/ ACCESS.2020.3035585

Wang, J., Hua, Z., Chen, Q., Xia, M., and Liu, W. (2020b). “Optimal Scheduling for Integrated Energy System Considering Shiftable Loads and Thermal Energy Storage of Building," in 2020 IEEE 4th Conference on Energy Internet and Energy System Integration (EI2) (IEEE), 1599-1603. doi:10.1109/EI250167.2020.9346871

Xiaojin Zhao, X., Yun Zhou, Y., Jingyang Yun, J., Peichao Zhang, P., Zheng Yan, Z., and Donghan Feng, D. (2019a). "Modelling and Operation Optimization of Electricity-Gas-Heat Integrated Energy System Considering Demand Response and Wind Penetration," in 8th Renewable Power Generation Conference (RPG 2019) (Shanghai, China: The Institution of Engineering and Technology), 1-8. doi:10.1049/cp.2019.0322

Xue, X., Ai, X., Fang, J., Yao, W., Wen, J., Shuai, H., et al. (2020). "Real-time Energy Management for the Integrated Heat and Power System Using Approximate Dynamic Programming," in 2020 IEEE Power Energy Society General Meeting (PESGM) (IEEE), 1-5. doi:10.1109/ PESGM41954.2020.9281887

Yang, H., Li, M., Jiang, Z., and Zhang, P. (2020). Multi-Time Scale Optimal Scheduling of Regional Integrated Energy Systems Considering Integrated
Demand Response. IEEE Access 8, 5080-5090. doi:10.1109/ ACCESS.2019.2963463

Yu, S., Yan, S., Liu, X., Liu, B., Xiong, C., and Liu, J. (2020). "Day-ahead Optimal Dispatch of Integrated Energy System Considering Wind Power Forecasting and Integrated Demand Response," in 2020 IEEE 1st China International Youth Conference on Electrical Engineering (CIYCEE) (IEEE), 1-7. doi:10.1109/ CIYCEE49808.2020.9332688

Zahedi, A. (1994). "Energy, People, Environment". Development of an Integrated Renewable Energy and Energy Storage System, an Uninterruptible Power Supply for People and for Better Environment. Proc. IEEE Int. Conf. Syst. Man Cybernetics 3, 2692-2695. doi:10.1109/ICSMC.1994.400279

Zeng, S., Wang, L., Chen, D., and Li, Y. (2017). "The Integrated Operation of Multiple Energy Carrier Used to Enhance the Reliability of the Integrated Energy System," in 2017 IEEE Conference on Energy Internet and Energy System Integration (EI2) (IEEE), 1-5. doi:10.1109/EI2.2017.8245575

Zhang, K., Zhou, B., Or, S. W., Li, C., Chung, C. Y., and Voropai, N. I. (2021). "Optimal Coordinated Control of Multi-Renewable-To-Hydrogen Production System for Hydrogen Fueling Stations," in IEEE Transactions on Industry Applications (IEEE), 1. doi:10.1109/TIA.2021.3093841

Zhang, N., Liu, L., Liu, K., and Mao, J. (2019). “A Novel Integrated Power-Gas-Heat System Planning Model Considering Energy Sources, Demand Response, Storage and Energy Converters," in 2019 IEEE 3rd International Electrical and Energy Conference (CIEEC) (IEEE), 500-504. doi:10.1109/ CIEEC47146.2019.CIEEC-2019211

Zhang, Q., and Wei, Q. (2020). "Optimized Configuration of Integrated Energy System Considering the Access and Operation of Renewable Energy," in 2020 IEEE 4th Conference on Energy Internet and Energy System Integration (EI2) (IEEE), 2470-2476. doi:10.1109/EI250167.2020.9346859

Zhao, W., Liao, Q., Xie, W., Zou, Z., Xu, D., and Liu, W. (2018). “CCHP Capacity Optimization with User Demand Characteristics," in 2018 International Conference on Power System Technology (POWERCON) (IEEE), 1687-1693. doi:10.1109/POWERCON.2018.8602112

Zhao, Z., Guo, J., Lai, C. S., Xiao, H., Zhou, K., and Lai, L. L. (2021). Distributed Model Predictive Control Strategy for Islands Multimicrogrids Based on Noncooperative Game. IEEE Trans. Ind. Inf. 17, 3803-3814. doi:10.1109/ TII.2020.3013102

Zhao, Z., Yang, P., Wang, Y., Xu, Z., and Guerrero, J. M. (2019b). Dynamic Characteristics Analysis and Stabilization of PV-Based Multiple Microgrid Clusters. IEEE Trans. Smart Grid 10, 805-818. doi:10.1109/TSG.2017.2752640

Conflict of Interest: Authors PL, FZ, XM, and SY were employed by the company Digital Grid Research Institute of China Southern Power Grid.

The remaining authors declare that the research was conducted in the absence of any commercial or financial relationships that could be construed as a potential conflict of interest.

Publisher's Note: All claims expressed in this article are solely those of the authors and do not necessarily represent those of their affiliated organizations, or those of the publisher, the editors and the reviewers. Any product that may be evaluated in this article, or claim that may be made by its manufacturer, is not guaranteed or endorsed by the publisher.

Copyright $\odot 2021 \mathrm{Li}$, Zhang, Ma, Yao, Zhong, Yang, Zhao, Lai and Lai. This is an open-access article distributed under the terms of the Creative Commons Attribution License (CC BY). The use, distribution or reproduction in other forums is permitted, provided the original author(s) and the copyright owner(s) are credited and that the original publication in this journal is cited, in accordance with accepted academic practice. No use, distribution or reproduction is permitted which does not comply with these terms. 